\title{
Article \\ Enmein Decreases Synaptic Glutamate Release and Protects against Kainic Acid-Induced Brain Injury in Rats
}

\author{
Cheng-Wei Lu ${ }^{1,2,+}$, Yu-Chen Huang ${ }^{1,+}$, Kuan-Ming Chiu ${ }^{3,4,5}$, Ming-Yi Lee ${ }^{3} \mathbb{D}$, Tzu-Yu Lin ${ }^{1,2, *}$ \\ and Su-Jane Wang 6,7,*(D)
}

check for

updates

Citation: Lu, C.-W.; Huang, Y.-C.; Chiu, K.-M.; Lee, M.-Y.; Lin, T.-Y.; Wang, S.-J. Enmein Decreases Synaptic Glutamate Release and Protects against Kainic Acid-Induced Brain Injury in Rats. Int. J. Mol. Sci. 2021, 22, 12966. https://doi.org/ $10.3390 /$ ijms222312966

Academic Editors: Hye Gwang Jeong Hwi-Yeol Yun and Hangun Kim

Received: 8 November 2021

Accepted: 29 November 2021

Published: 30 November 2021

Publisher's Note: MDPI stays neutral with regard to jurisdictional claims in published maps and institutional affiliations.

Copyright: (c) 2021 by the authors. Licensee MDPI, Basel, Switzerland. This article is an open access article distributed under the terms and conditions of the Creative Commons Attribution (CC BY) license (https:// creativecommons.org/licenses/by/ $4.0 /)$.
1 Department of Anesthesiology, Far-Eastern Memorial Hospital, New Taipei City 22060, Taiwan; drluchengwei@gmail.com (C.-W.L.); yuchenhuang@msn.com (Y.-C.H.)

2 Department of Mechanical Engineering, Yuan Ze University, Taoyuan 32003, Taiwan

3 Division of Cardiovascular Surgery, Cardiovascular Center, Far-Eastern Memorial Hospital, New Taipei 22060, Taiwan; chiu9101018@gmail.com (K.-M.C.); mingyi.lee@gmail.com (M.-Y.L.)

4 Department of Nursing, Asia Eastern University of Science and Technology, New Taipei City 22060, Taiwan

5 Department of Photonics Engineering, Yuan Ze University, Taoyuan 32003, Taiwan

6 Research Center for Chinese Herbal Medicine, College of Human Ecology, Chang Gung University of Science and Technology, Taoyuan 33303, Taiwan

7 School of Medicine, Fu Jen Catholic University, New Taipei City 24205, Taiwan

* Correspondence: drlin1971@gmail.com (T.-Y.L.); med0003@mail.fju.edu.tw (S.-J.W.)

+ Equal contribution to Cheng-Wei Lu and Yu-Chen Huang.

Abstract: This study investigated the effects of enmein, an active constituent of Isodon japonicus Hara, on glutamate release in rat cerebrocortical nerve terminals (synaptosomes) and evaluated its neuroprotective potential in a rat model of kainic acid (KA)-induced glutamate excitotoxicity. Enmein inhibited depolarization-induced glutamate release, FM1-43 release, and $\mathrm{Ca}^{2+}$ elevation in cortical nerve terminals but had no effect on the membrane potential. Removing extracellular $\mathrm{Ca}^{2+}$ and blocking vesicular glutamate transporters, $\mathrm{N}$ - and $\mathrm{P} / \mathrm{Q}$-type $\mathrm{Ca}^{2+}$ channels, or protein kinase $\mathrm{C}$ (PKC) prevented the inhibition of glutamate release by enmein. Enmein also decreased the phosphorylation of PKC, PKC- $\alpha$, and myristoylated alanine-rich C kinase substrates in synaptosomes. In the KA rat model, intraperitoneal administration of enmein $30 \mathrm{~min}$ before intraperitoneal injection of KA reduced neuronal cell death, glial cell activation, and glutamate elevation in the hippocampus. Furthermore, in the hippocampi of KA rats, enmein increased the expression of synaptic markers (synaptophysin and postsynaptic density protein 95) and excitatory amino acid transporters 2 and 3 , which are responsible for glutamate clearance, whereas enmein decreased the expression of glial fibrillary acidic protein (GFAP) and CD11b. These results indicate that enmein not only inhibited glutamate release from cortical synaptosomes by suppressing $\mathrm{Ca}^{2+}$ influx and PKC but also increased KA-induced hippocampal neuronal death by suppressing gliosis and decreasing glutamate levels by increasing glutamate uptake.

Keywords: enmein; glutamate release; $\mathrm{Ca}^{2+}$ influx; glutamate transporter; neuroprotection; antiinflammation; excitotoxicity

\section{Introduction}

Glutamate is an excitatory neurotransmitter that is involved in many physiological functions of the brain [1,2]. However, excess glutamate in the synaptic cleft causes an overactivation of glutamate receptors, which increases the intracellular $\mathrm{Ca}^{2+}$ concentration and results in reactive oxygen species generation, oxidative stress, and mitochondrial dysfunction, ultimately resulting in neuronal cell damage or death. In addition, changes in glutamate levels can disrupt glutamatergic neurotransmission, which can be a cause of neurological diseases such as stroke, epilepsy, traumatic brain injury, and psychiatric and neurodegenerative diseases $[3,4]$. Therefore, preventing glutamate excitotoxicity in the 
nervous system is considered to be a promising target for clinical trials to develop drugs to treat these neurological disorders [5-7].

Natural products are an invaluable source for novel drug research because of their safety and various biological functions [8]. The search for and discovery of active components in medicinal plants is a popular topic in pharmaceutical chemistry. In particular, many natural constituents from medicinal plants have been reported to reduce synaptic glutamate release and thereby protect neurons, including baicalein, a flavonoid from Scutellaria baicalensis; echinacoside, a phenylethanoid glycoside from Herba Cistanche; 11-keto- $\beta$-boswellic acid, a triterpenoid from Boswellia serrata; and asiatic acid, a triterpene from Centella asiatica [9-13]. Therefore, natural products that inhibit central glutamatergic neurotransmission may offer a possible therapeutic option for treating neurological diseases [13]. Enmein is a diterpenoid derived from the plant Isodon japonicus Hara that is widely used in dietary supplements and folk medicine [14]. Enmein has antibacterial [15], anti-inflammatory [16], antitumor [17], and immunosuppressive effects [18]. There is currently no data to support the effects of enmein on the central glutamate system. The aim of the present study was to evaluate: (1) whether enmein has an effect on glutamate release in the cerebral cortex nerve terminals (synaptosomes) of rats; and (2) whether enmein protects against excitotoxicity caused by kainic acid (KA), a glutamate analog [18]. In the synaptosomal model, the effects of enmein on glutamate release, membrane potential, $\mathrm{Ca}^{2+}$ concentrations, and the $\mathrm{Ca}^{2+}$-activated protein kinase $\mathrm{C}$ (PKC) signaling cascade were evaluated. In the KA rat model of excitotoxicity, we evaluated the effects of enmein pretreatment on neuronal damage, glutamate elevation, glial cell activation, and the expression of excitatory amino acid transporters (EAATs) 2 and 3, which are responsible for glutamate clearance, synaptic plasticity-related proteins, synaptophysin and postsynaptic density protein 95 (PSD95), and hippocampal glial fibrillary acidic protein (GFAP) and CD11b.

\section{Results}

\subsection{The Influence of Enmein on Glutamate Release in Rat Cerebrocortical Nerve Terminals}

To investigate the influence of enmein on glutamate release, isolated nerve terminals were depolarized with the $\mathrm{K}^{+}$channel blocker 4-Aminopyridine (4-AP), which increases $\mathrm{Ca}^{2+}$ influx and glutamate release [19]. Figure 1A shows that in synaptosomes incubated in the presence of $1 \mathrm{mM} \mathrm{CaCl}_{2}, 4-\mathrm{AP}(1 \mathrm{mM})$ evoked significant glutamate release. Incubation with enmein $(30 \mu \mathrm{M})$ for $10 \mathrm{~min}$ before 4-AP administration did not have a significant effect on the basal release of glutamate but markedly reduced the glutamate release mediated by $4-\mathrm{AP}[\mathrm{t}(8)=3.9, p<0.001$ vs. the control group]. Enmein inhibited glutamate release concentration-dependently, with a maximum inhibition of $56.3 \% \pm 1.6 \%$ produced at $30 \mu \mathrm{M}$ and a half maximal effective concentration of $8 \mu \mathrm{M}$ (Figure 1B). In addition, in extracellular $\mathrm{Ca}^{2+}$-free medium containing EGTA, glutamate release evoked by 4-AP from synaptosomes was reduced to approximately $67 \%$ of that detected in the presence of external $\mathrm{Ca}^{2+}$ $[\mathrm{t}(8)=0.8, p<0.001]$. Notably, this 4 -AP-evoked $\mathrm{Ca}^{2+}$-independent release was not affected by prior enmein addition ( $p=0.24$ vs. $\mathrm{Ca}^{2+}$-free group; Figure $1 \mathrm{C}$ ). Similarly, enmein failed to cause significant inhibition in the presence of bafilomycin A1, a vacuolar $\mathrm{H}^{+}$ATPase inhibitor $[\mathrm{F}(3,16)=1467.1 ; p=0.16$ vs. bafilomycin A1-treated group, Figure $1 \mathrm{C}$, inset]. Bafilomycin A1 alone did reduce 4-AP-evoked glutamate release $(p<0.001)$. Furthermore, the effect of enmein on 4-AP-triggered exocytosis was evaluated by analyzing the reduction in FM1-43 fluorescence intensity [20]. As shown in Figure 1D, 4-AP (1 mM) caused a decrease in FM1-43 fluorescence in the presence of $\mathrm{CaCl}_{2}$. Preincubating synaptosomes with enmein for 10 min significantly reduced the 4-AP-induced decrease in FM1-43 fluorescence $[\mathrm{t}(8)=6.3 ; p<0.001]$. These results suggest that the inhibition of glutamate release by enmein is due to a decrease in the $\mathrm{Ca}^{2+}$-dependent exocytotic mechanism. 
A

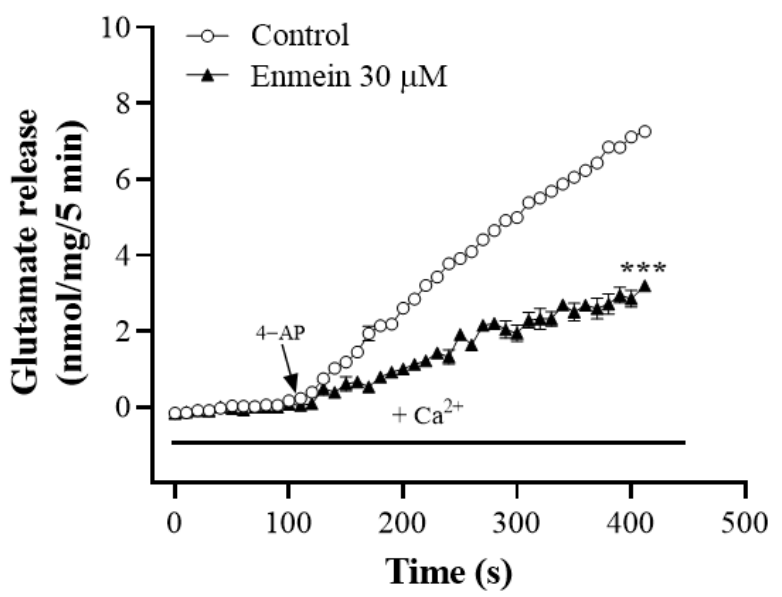

C

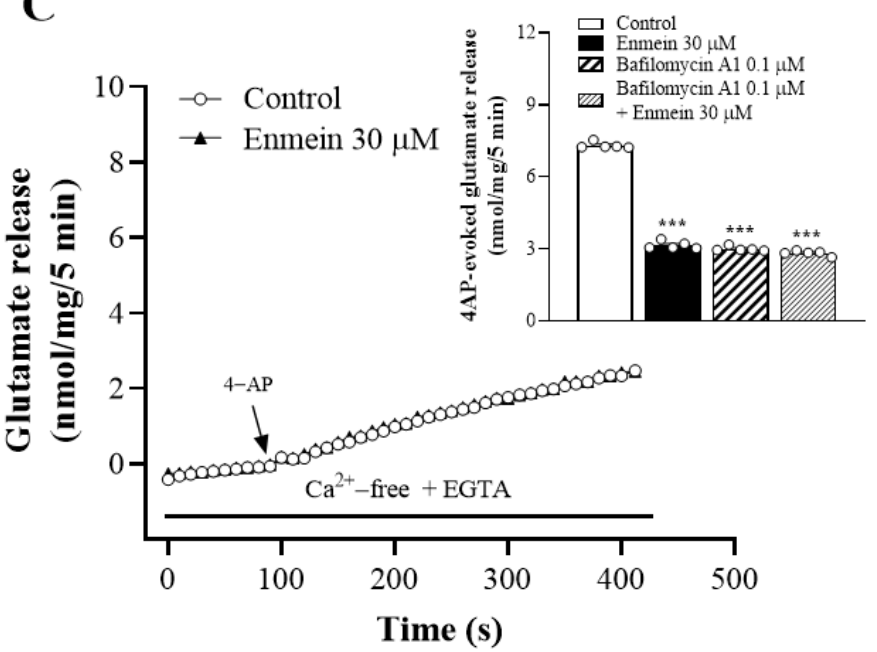

B

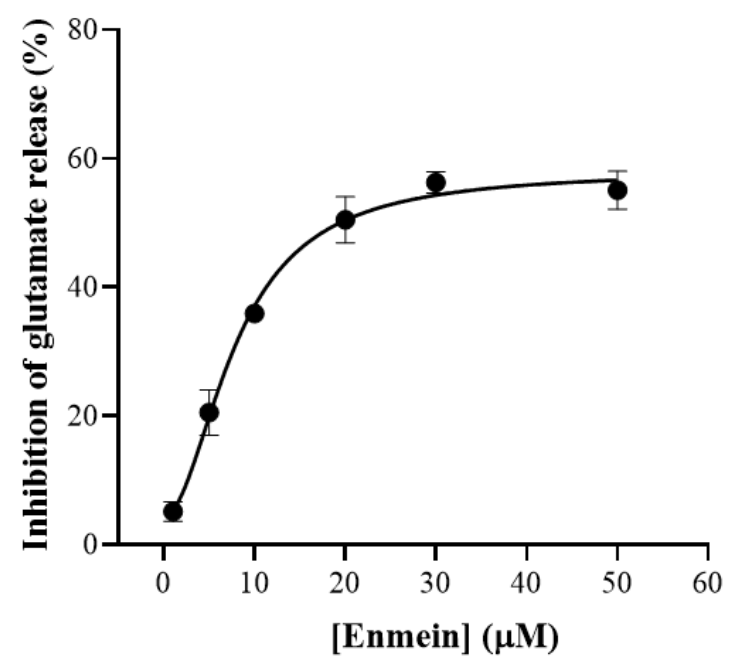

D

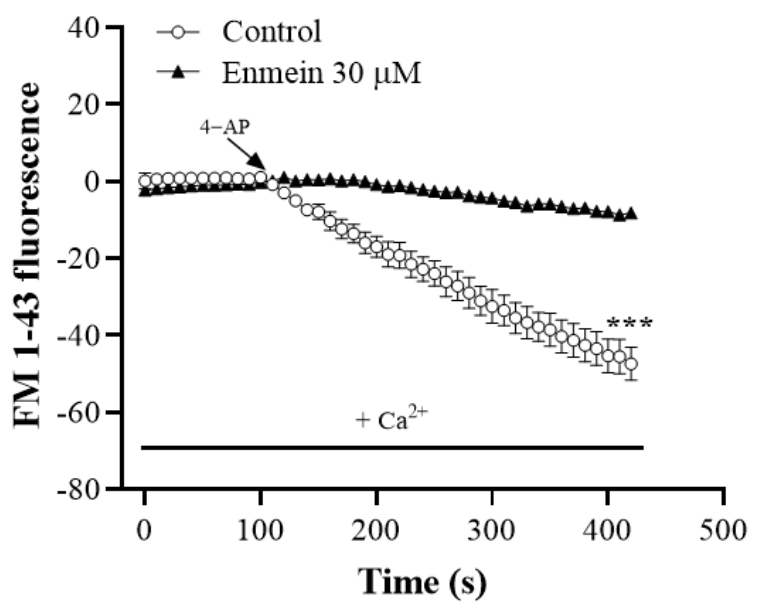

Figure 1. Effect of enmein on 4-AP-evoked glutamate release from rat cerebrocortical synaptosomes. (A) 4-AP (1 mM)evoked glutamate release from synaptosomes incubated in the presence of $1.2 \mathrm{mM} \mathrm{CaCl}_{2}$, and in the absence (control) or presence of enmein. (B) Concentration-response curve of decreases in 4-AP-evoked glutamate release in the presence enmein. (C) Extracellular $\mathrm{Ca}^{2+}$-free solution containing EGTA $(300 \mu \mathrm{M})$ on the action of enmein. Inset, glutamate release was evoked by 4-AP in the absence (control) or presence of bafilomycin A1, or bafilomycin A1 + enmein. (D) The release of FM1-43 was evoked by 4-AP in the absence (control) or presence of enmein. Enmein was added 10 min before the addition of 4 -AP, and other drugs were added $10 \mathrm{~min}$ before this. Data are the mean $\pm \operatorname{SEM}\left(n=5\right.$ per group). ${ }^{* *}, p<0.001$ vs. control group.

2.2. The Influence of Enmein on Cytosolic Free $\mathrm{Ca}^{2+}$ Concentration $\left(\left[\mathrm{Ca}^{2+}\right]_{C}\right)$ and Synaptosomal Plasma Membrane Potential

As shown in Figure 2A, fluorometric experiments performed in synaptosomes containing Fura-2-acetoxymethyl ester (Fura-2-AM) and depolarized with $1 \mathrm{mM}$ 4-AP showed that the rise of $\left[\mathrm{Ca}^{2+}\right]_{C}$ above basal levels was significantly reduced in the presence of enmein $[\mathrm{t}(8)=9.1, p<0.001$ vs. control group]. However, enmein had no significant effect on basal $\left[\mathrm{Ca}^{2+}\right]_{\mathrm{C}}$ levels $[\mathrm{t}(8)=1.4, p=0.21 \mathrm{vs}$. control group]. In addition, we examined the effect of enmein on synaptosomal plasma membrane potential by using the membrane potential-sensitive dye $\mathrm{DiSC}_{3}(5)$. As shown in Figure 2B, 4-AP (1 mM) caused an increase in $\mathrm{DiSC}_{3}(5)$ fluorescence. Preincubation of synaptosomes with enmein had no effect on the resting plasma membrane potential $[\mathrm{t}(8)=0.25, p=0.8 \mathrm{vs}$. control group]; however, 
the 4-AP-induced elevation of $\mathrm{DiSC}_{3}(5)$ fluorescence was significantly decreased in the presence of enmein $[\mathrm{t}(8)=1.8, p=0.1 \mathrm{vs}$. control group].

A

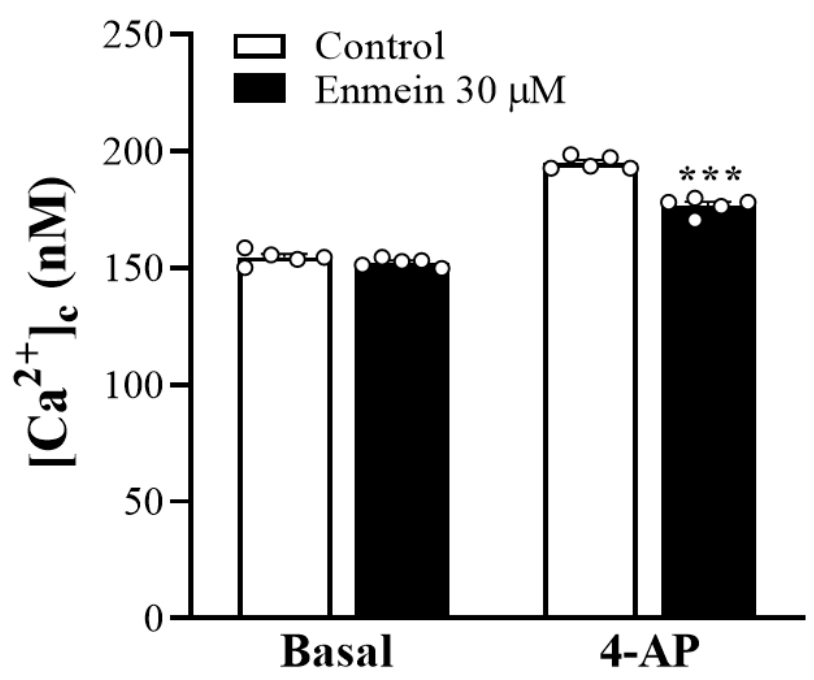

B

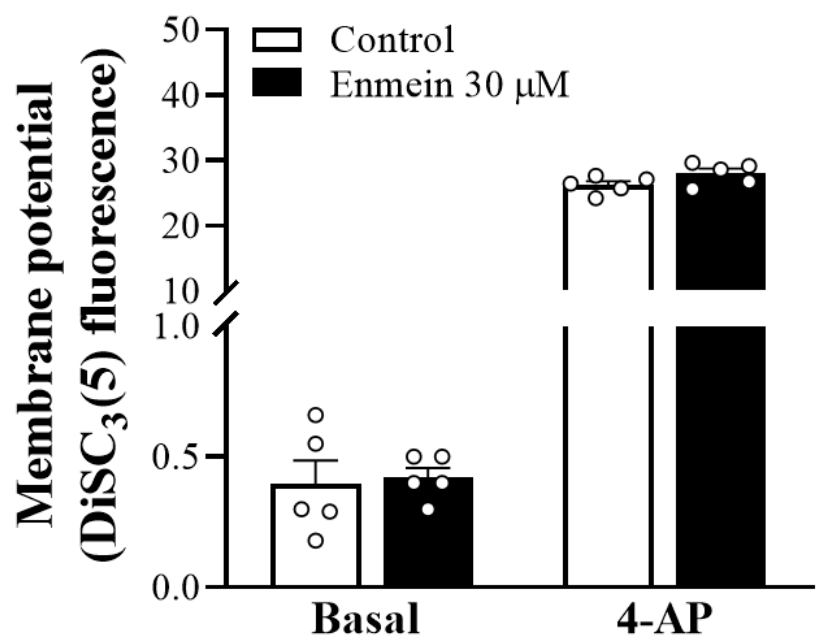

Figure 2. Effect of enmein on $\left[\mathrm{Ca}^{2+}\right]_{\mathrm{C}}(\mathbf{A})$ and the synaptosomal membrane potential $(\mathbf{B})$. Enmein was added 10 min before the addition of 4 -AP $(1 \mathrm{mM})$. Data are presented as mean \pm SEM. ( $n=5$ per group). ${ }^{* * *}, p<0.001$ vs. control group.

2.3. The Reduction in $\mathrm{N}$ - and P/Q-type $\mathrm{Ca}^{2+}$ Channel Activities Is Involved in the Enmein-Induced Inhibition of Glutamate Release

In rat cerebrocortical nerve terminal preparations, $\mathrm{N}$ - and $\mathrm{P} / \mathrm{Q}$-type voltage-dependent $\mathrm{Ca}^{2+}$ channels (VDCCs) promoted glutamate release [21]. Similarly, when VDCCs were blocked with $2 \mu \mathrm{M} \omega$-conotoxin GVIA ( $\omega$-CgTX GVIA) (N-type) or $200 \mathrm{nM}$ - $\omega$-agatoxin IVA ( $\omega$-AgTX IVA) (P/Q-type), 4-AP-evoked glutamate release was largely reduced $[\mathrm{F}(3,23)=461.3 ; p<0.001$ vs. control group $]$, and the inhibitory effect of enmein was not detectable ( $p=0.99$ vs. $\omega$-CgTX GVIA and $\omega$-AgTX IVA-treated group; Figure 3 ). Similar results were obtained using $1 \mu \mathrm{M} \omega$-CgTX MVIIC, a wide-spectrum blocker of $\mathrm{N}-, \mathrm{P}-$, and Q-type $\mathrm{Ca}^{2+}$ channels. $\omega$-CgTX MVIIC alone reduced 4-AP-evoked glutamate release $[F(3,23)=381.8 ; p<0.001$ vs. control group]. However, the release measured in the presence of both $\omega$-CgTX MVIIC and enmein was similar to that obtained in the presence of only $\omega$-CgTX MVIIC ( $p=0.47)$. In addition, we examined the effect of enmein on $15 \mathrm{mM}$ $\mathrm{KCl}$-evoked glutamate release, a mechanism that involves $\mathrm{Ca}^{2+}$ influx primarily via VDCC openings [22]. As shown in Figure 3, enmein also significantly inhibited KCl-evoked glutamate release $[\mathrm{t}(8)=17.9, p<0.001$ vs. control].

\subsection{The Suppression of PKC/MARCKS Pathways Is Linked to Enmein-Induced Inhibition of Glutamate Release}

Increased $\mathrm{Ca}^{2+}$ influx in nerve terminals has been shown to enhance PKC activation and subsequently enhance glutamate release [23]. We therefore investigated whether the inhibition of $\mathrm{Ca}^{2+}$ influx caused by enmein decreased PKC activity and thus decreased glutamate release. As shown in Figure 4A, $10 \mu \mathrm{M}$ isindolylmaleimide I (GF109203X), a general PKC inhibitor, reduced 4-AP-evoked glutamate release $[\mathrm{F}(3,25)=491.7, p<0.001$ vs. control group]. When GF109203X was present, enmein did not significantly inhibit glutamate release ( $p=0.98$ vs. GF109203X-treated group). Similarly, in the presence of $1 \mu \mathrm{M}, 5,6,7,13$-tetrahydro-13-methyl-5-oxo-12H-indolo[2,3-a]pyrrolo[3,4-c]carbazole-12propanenitrile (Go6976), an inhibitor of conventional $\mathrm{Ca}^{2+}$-dependent PKC- $\alpha$ isozymes, 4 -AP-evoked glutamate release was reduced $[\mathrm{F}(3,23)=465.9 ; p<0.001$ vs. control group] In addition, enmein did not reduce 4-AP-evoked glutamate release when Go6976 was present ( $p=0.99$ vs. Go6976-treated group; Figure 4A). Furthermore, Figure 4B shows that synaptosome depolarization with $1 \mathrm{mM} 4$-AP in the presence of $1.2 \mathrm{mM} \mathrm{CaCl}_{2}$ increased the 
phosphorylation of PKC $[\mathrm{F}(2,12)=874.5 ; p<0.001$ vs. control group $], \operatorname{PKC}-\alpha[\mathrm{F}(2,12)=39.9$; $p<0.001$ vs. control group], and myristoylated alanine-rich $C$ kinase substrate (MARCKS), a key presynaptic substrate for PKC $[\mathrm{F}(2,12)=142.1 ; p<0.001 \mathrm{vs}$. control group $]$. These 4-APinduced phosphorylation elevations were markedly decreased by the presence of enmein ( $p<0.05$ vs. the 4-AP-treated group). 4-AP did not affect PKC and PKC- $\alpha$ expression [PKC, $\mathrm{F}(2,12)=0.44 ; p=0.95$ vs. control group; PKC- $\alpha, \mathrm{F}(2,12)=0.69 ; p=0.99$ vs. control group] (Figure $4 \mathrm{~B}, \mathrm{C}$ ).

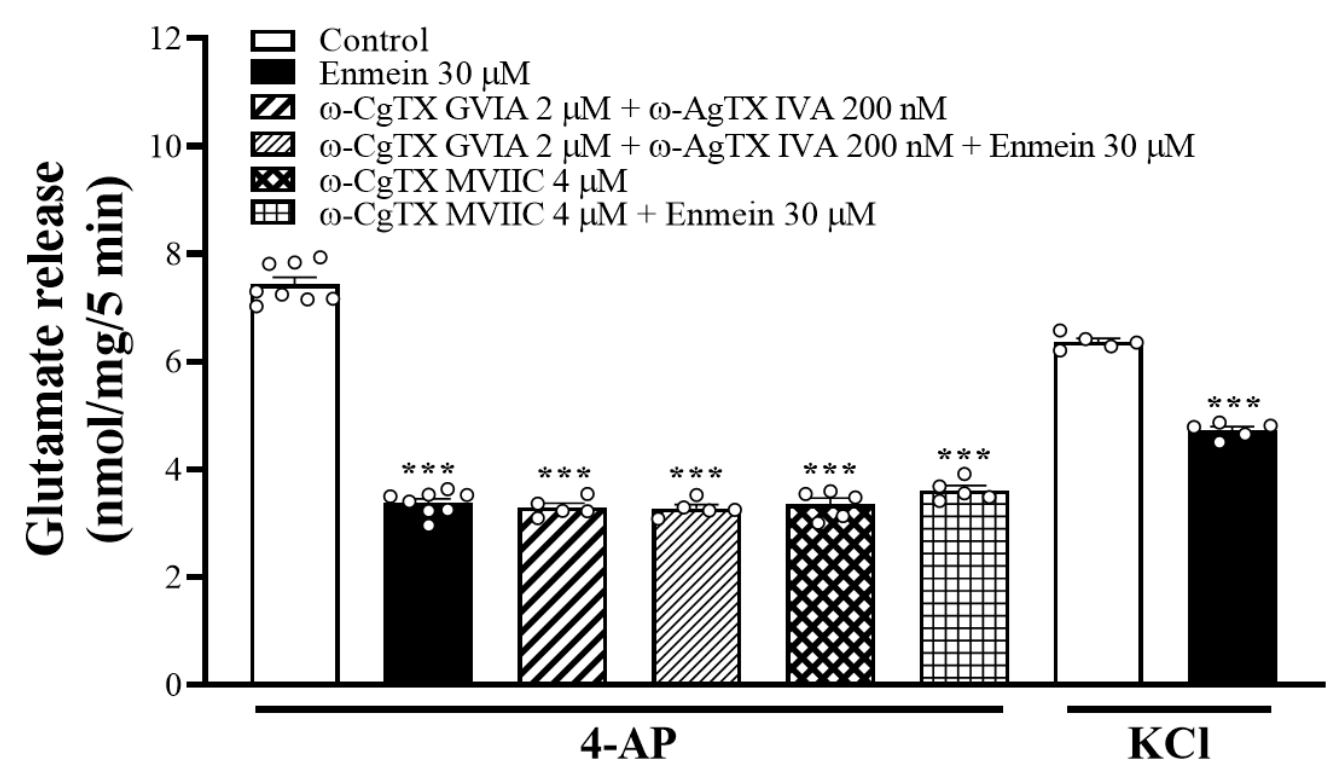

Figure 3. Blockade of $\mathrm{N}$ - and $\mathrm{P} / \mathrm{Q}$-type $\mathrm{Ca}^{2+}$ channels abolishes enmein inhibition of glutamate release. Glutamate release was evoked by $1 \mathrm{mM} 4-\mathrm{AP}$ in the absence (control) or presence of enmein, $\omega$-CgTX GVIA + $\omega$-AgTX IVA, or $\omega$-CgTX GVIA + $\omega$-AgTX IVA + enmein. Effect of enmein on the release of glutamate evoked by $15 \mathrm{mM} \mathrm{KCl}$ was also examined. Enmein was added $10 \mathrm{~min}$ before the addition of 4-AP, and other drugs were added $10 \mathrm{~min}$ before this. Data are presented as mean \pm S.E.M. $\left(n=5\right.$ per group). ${ }^{* *}, p<0.001$ vs. control group.

\subsection{The Influence of Enmein on Neuronal Death in the Hippocampi of KA Rats}

The neuroprotective effect of enmein was evaluated in a rat model of KA-induced glutamate excitotoxicity. As shown in Figure 5A, rats were given either enmein or dimethylsulfoxide (DMSO) intraperitoneally (i.p.) $30 \mathrm{~min}$ before KA injection (i.p.). Nissl staining was performed to examine neuronal survival in the hippocampus $72 \mathrm{~h}$ after KA injection. Compared to the control group, in the KA-treated group, significant neuronal loss in the CA1 and CA3 regions was observed [CA1, $\mathrm{F}(2,6)=483.9, p<0.001 ; \mathrm{CA} 3, \mathrm{~F}(2,6)=279.4$, $p<0.001$; Figure 5B]. However, in the enmein/KA-treated group, the number of surviving neurons in the CA1 and CA3 regions was significantly higher than that in the KA-treated group ( $p<0.05$; Figure $5 \mathrm{~B}$ ). In addition, the histofluorescence findings of neuronal nuclear protein (NeuN) (a specific neuron marker) were consistent with those of Nissl staining. Compared to that in the control group, the number of NeuN-positive neurons in the KA-treated group were significantly decreased in both the CA1 and CA3 regions [CA1, $\mathrm{F}(2,6)=684.3, p<0.001 ; \mathrm{CA} 3, \mathrm{~F}(2,6)=642.9, p<0.001]$. Compared to that in the KA-treated group, the number of NeuN-positive neurons in the enmein/KA-treated group was increased in both the CA1 and CA3 regions ( $p<0.05$; Figure 5A,C). Similarly, the expression of NeuN in the hippocampus was significantly lower in the KA-treated group than in the control group $[\mathrm{F}(2,9)=318.7, p<0.001]$. In contrast, the expression of NeuN in the hippocampus was higher in the enmein/KA-treated group than in the KA-treated group $(p<0.05$; Figure 5D). 
A

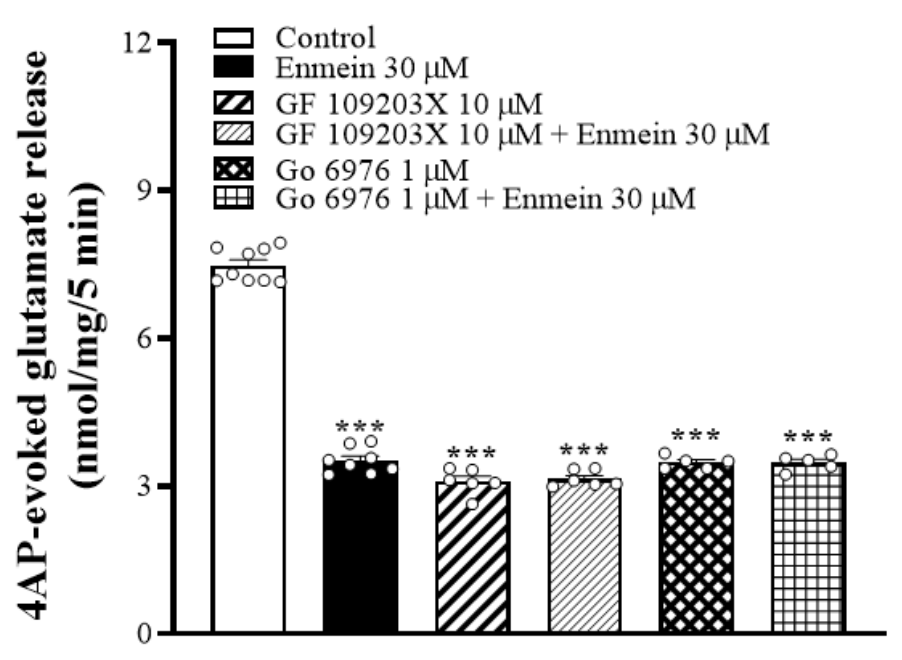

C

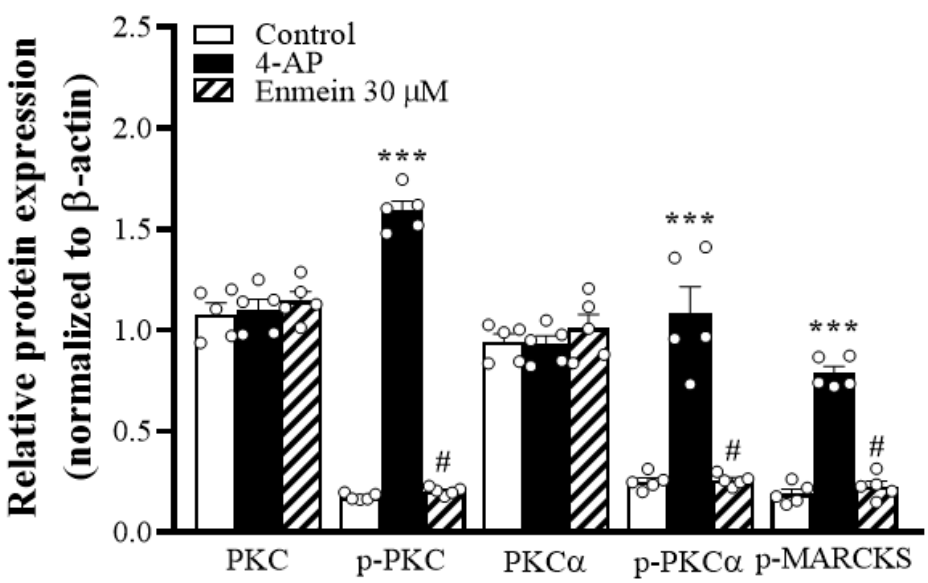

B

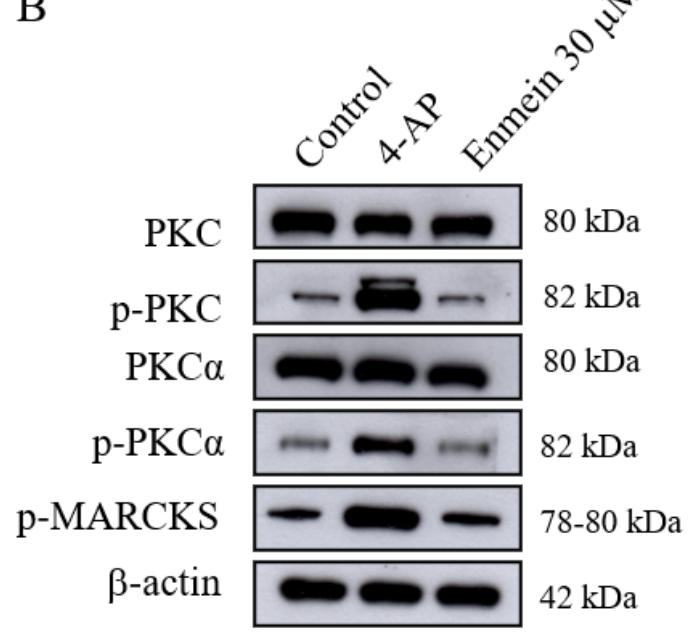

Figure 4. Enmein-mediated inhibition of glutamate release is linked to the suppression of PKC-dependent pathway. (A) Glutamate release was evoked by $1 \mathrm{mM} 4$-AP in the absence (control) or presence of enmein, GF109203X (a PKC inhibitor), Go6976 (a PKC $\alpha$ inhibitor), GF109203X + enmein, or Go6976 + enmein. (B) The expression of PKC, phospho-PKC (p-PKC), PKC $\alpha$, phospho-PKC- $\alpha$ (p-PKC- $\alpha)$, and MARCKS was detected in synaptosomal lysates by Western blotting. (C) The quantification of protein expression of PKC, p-PKC, PKC $\alpha, \mathrm{p}-\mathrm{PKC} \alpha$, and MARCKS was normalized to $\beta$-actin. Enmein was added $10 \mathrm{~min}$ before the addition of $4-\mathrm{AP}$, and other drugs were added 20 min before this. Data are presented as mean \pm SEM. ( $n=5$ per group). ${ }^{* *}, p<0.001$ vs. control group. $\#, p<0.05$ vs. 4 -AP group.

2.6. The Influence of Enmein on Glutamate Elevation and the Expression of EAAT2 and EAAT3 in the Hippocampi of KA Rats

To elucidate the possible mechanism of the neuroprotective action of enmein in our study, glutamate concentrations-a key neurotransmitter in the central nervous system underlying the pathophysiology of neurological disorders-were determined $72 \mathrm{~h}$ after KA injection by high-performance liquid chromatography (HPLC). As shown in Figure 6A, glutamate concentrations in the hippocampi of the KA-treated group were significantly higher than in the control group $[\mathrm{F}(2,9)=164.7, p<0.001]$. In contrast, the hippocampal glutamate level of the enmein/KA-treated group was significantly lower than that of the KA-treated group ( $p<0.05$; Figure 6A). Excitatory amino acid transporters (EAATs), especially EAAT2 and EAAT3, are critical for maintaining low extracellular glutamate concentrations and preventing excitotoxicity [24-26]. To investigate how enmein reduced 
KA-induced glutamate elevation in the hippocampus, we examined the expression of EAAT2 and EAAT3 in the hippocampus. As shown in Figure 6B, Western blot analysis showed that EAAT2 and EAAT3 hippocampal levels were significantly lower in the KAtreated group than in the control group [EAAT2, $\mathrm{F}(2,9)=12.9, p<0.01$; EAAT3, $[\mathrm{F}(2,9)=30.5$, $p<0.001]$. However, in the enmein/KA-treated group, the expression levels of EAAT2 and EAAT3 were significantly higher than those in the KA-treated group $(p<0.05)$ (Figure 6B).

A
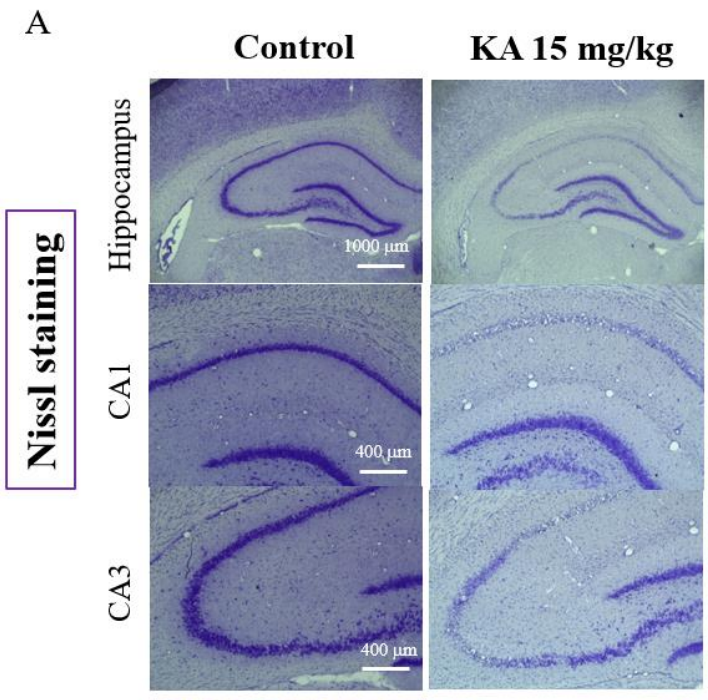

Enmein $50 \mathrm{mg} / \mathrm{kg}$
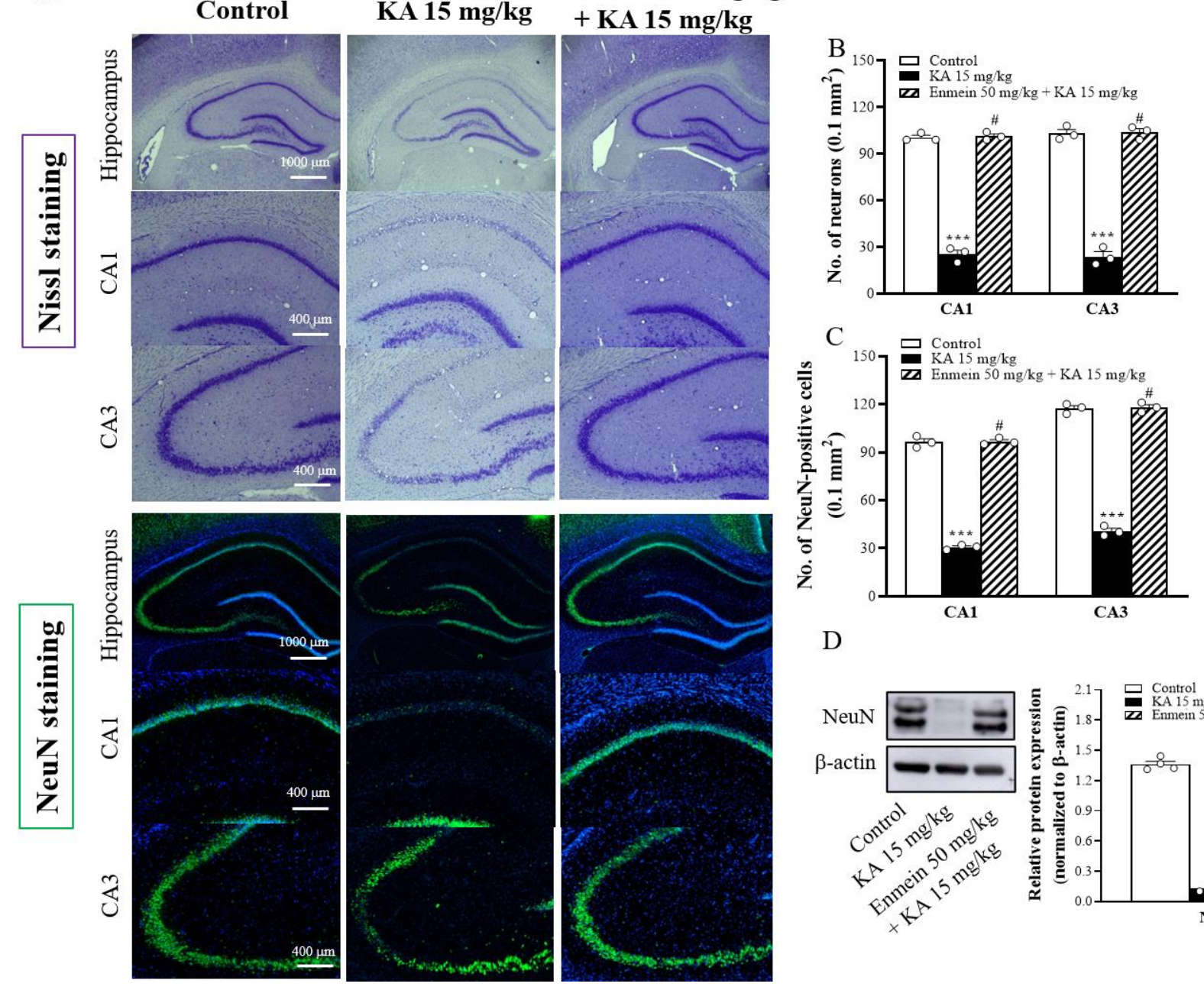

$\mathrm{D}$
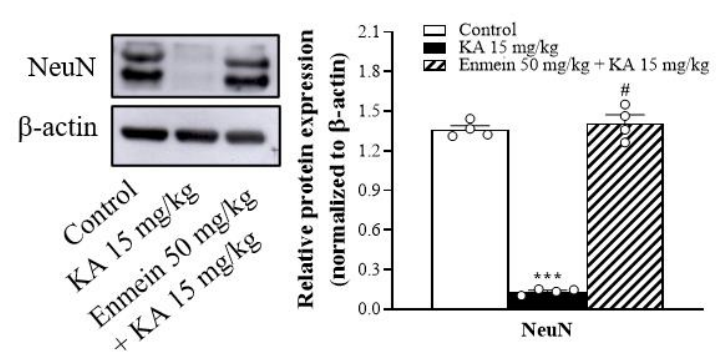

Figure 5. Effect of enmein pretreatment on the neuronal cell death in the hippocampus of rats with KA. (A) Representative images of Nissl and NeuN staining at $72 \mathrm{~h}$ after KA injection (i.p.). (B,C) Semiquantitative analysis of neurons and NeuNpositive cells in the CA1 and CA3 regions. (D) Western blot and semiquantitative analysis for NeuN in the hippocampus from differently treated groups. Data are presented as mean \pm SEM. $\left(n=3-4\right.$ rats for each group). ${ }^{* * *}, p<0.001$ vs. control group. \#, $p<0.05$ vs. KA group.

2.7. The Influence of Enmein on the Expression of Synaptic Marker Proteins (Synaptophysin and PSD95) in the Hippocampi of KA Rats

KA-induced neuronal toxicity has been shown to cause synaptic dysfunction $[27,28]$. The hippocampal levels of the synaptic marker proteins synaptophysin and PSD95 were evaluated $72 \mathrm{~h}$ after KA injection. As shown in Figure 7, Western blot analysis revealed that synaptophysin and PSD95 levels in the hippocampus were lower in the KA-treated group than in the control group [synaptophysin, $\mathrm{F}(2,9)=106.6, p<0.001$; PSD95, F(2,9) = 270.3, $p<0.001]$. Animals pretreated with enmein had significantly higher levels than the KAtreated group $(p<0.05)$. 

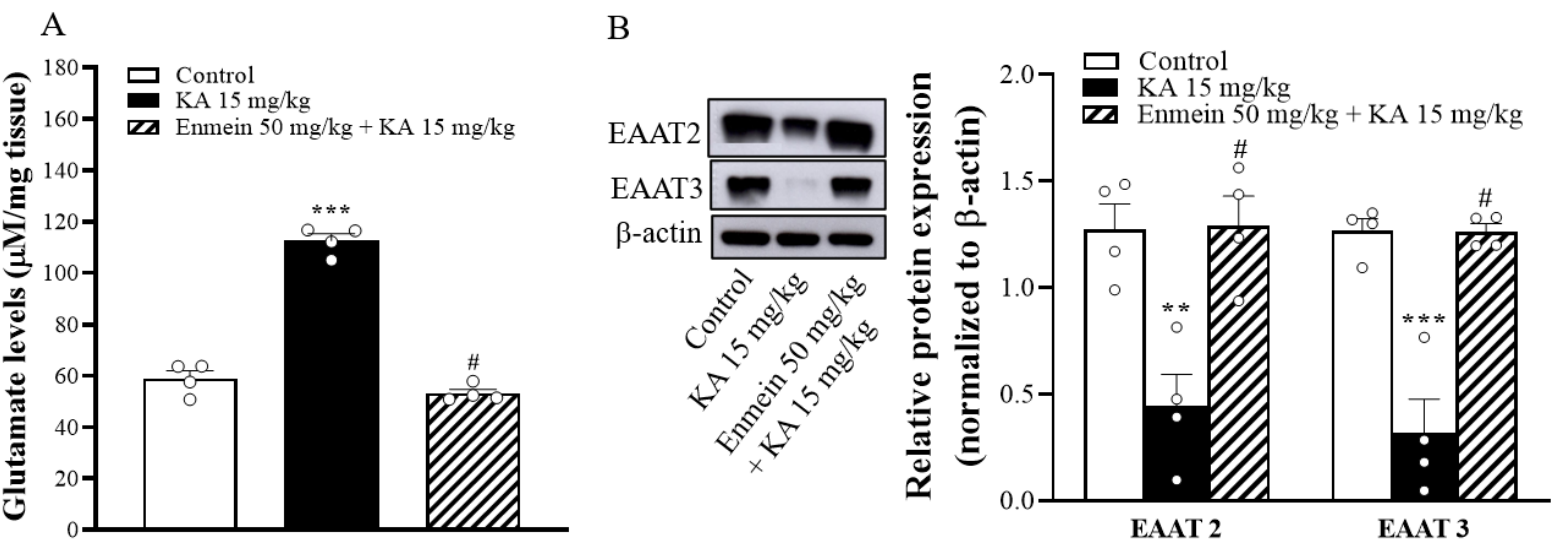

Figure 6. Effect of enmein pretreatment on the glutamate levels (A) and expression of EAAT2 and EAAT3 (B) in the hippocampus of rats with KA. The glutamate concentrations and EAAT2 and EAAT3 expression levels in the hippocampus were semiquantitative evaluated at $72 \mathrm{~h}$ after KA injection by high-performance liquid chromatography (HPLC) and Western blot, respectively. Data are presented as mean \pm SEM. ( $n=4$ rats for each group). ${ }^{* *}, p<0.01 ;{ }^{* * *}, p<0.001$ vs. control group. \#, $p<0.05$ vs. KA group.
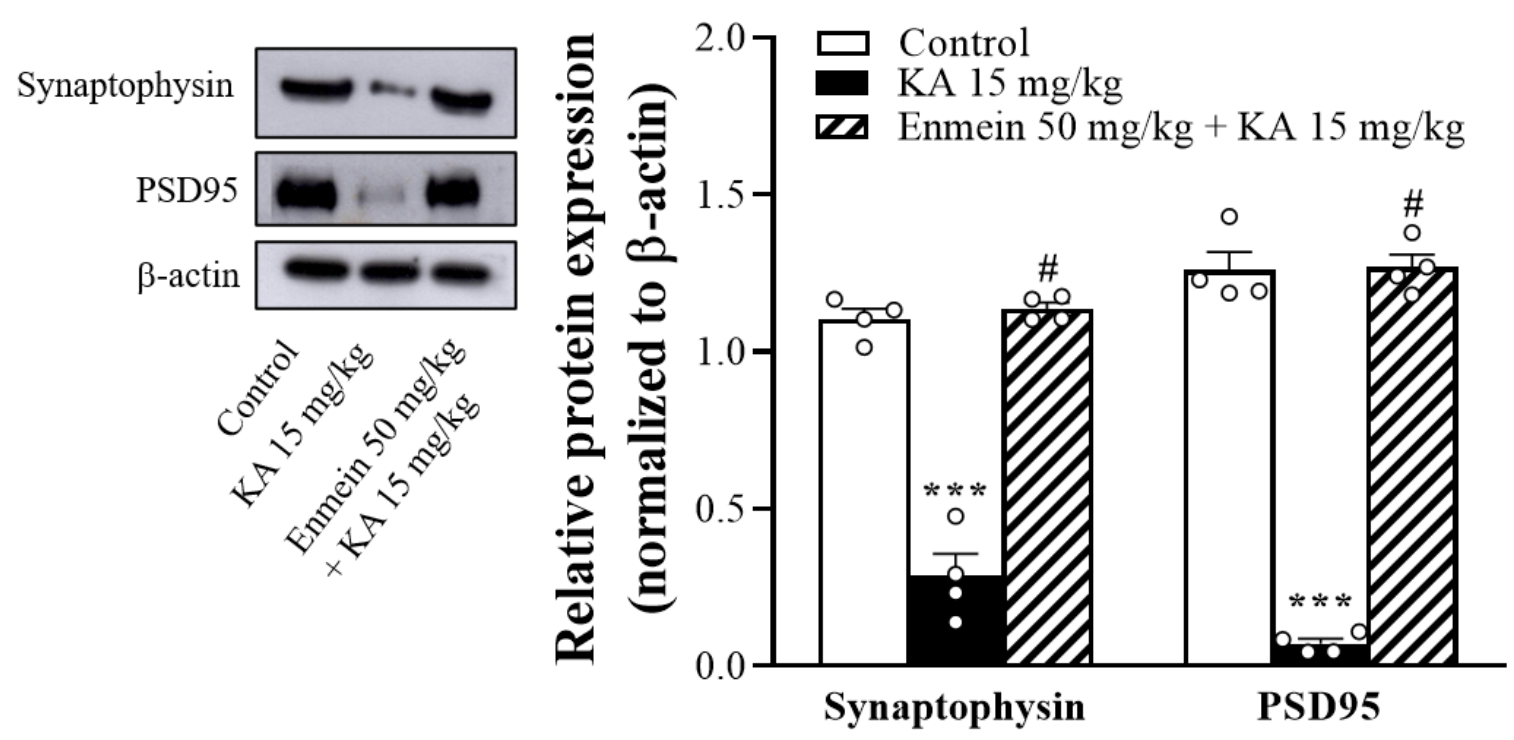

Figure 7. Effect of enmein on the expression levels of synaptophysin and PSD95 in the hippocampi of rats with KA. Western blot and semiquantitative analysis for PSD95 and synaptophysin in the hippocampus from differently treated groups. Data are presented as mean \pm SEM. ( $n=4$ rats for each group). ${ }^{* * *}, p<0.001$ vs. control group. $\#, p<0.05$ vs. KA group.

\subsection{The Influence of Enmein on the Activation of Glial Cells in the Hippocampi of KA Rats}

Accumulating evidence has suggested that KA-induced excitotoxicity enhances glial cell activation and promotes neuroinflammatory responses in the brain [29-31]. As an important neuroinflammation mediator, we analyzed the expression of GFAP (an astrocyte marker) and CD11b (a microglial marker) proteins in the hippocampal CA1 and CA3 regions of the experimental animals $72 \mathrm{~h}$ after KA injection. Compared to the control group, the KA-injected group displayed a significant induction of gliosis, as revealed by the increased expression of GFAP and CD11b proteins [GFAP, F(2,9) = 68.7, $p<0.001$; CD11b, $\mathrm{F}(2,9)=99.6, p<0.001$ ] (Figure 8A). Immunohistochemical analysis further confirmed the induction of gliosis in the brain slices of KA-injected rats, as illustrated by a higher number of GFAP-positive [CA1, F(2,6) =254.5, $p<0.001$; CA3, F(2,6) = 182.4, $p<0.001$ ] and Iba-1positive cells [CA1, $\mathrm{F}(2,6)=24.1, p<0.01 ; \mathrm{CA} 3, \mathrm{~F}(2,6)=74.3, p<0.001]$ in the hippocampal CA1 and CA3 regions than in the control group (Figure $8 \mathrm{~B}-\mathrm{D})$. In contrast, the expression of GFAP and Iba-1 in the hippocampus was lower in the enmein/KA-treated group than 
in the KA-treated group ( $p<0.05$; Figure $8 \mathrm{~A}$ ). The brain slices of the enmein/KA-treated group also had a reduced number of GFAP- and Iba-1-positive cells in the hippocampal CA1 and CA3 regions ( $p<0.05$ vs. KA group; Figure $8 \mathrm{~B}-\mathrm{D}$ ). These findings show that KA-induced excitotoxic damage is related to neuroinflammation and that pretreatment with enmein could protect the brain from the neuroinflammatory mediator associated with the pathological state.

A
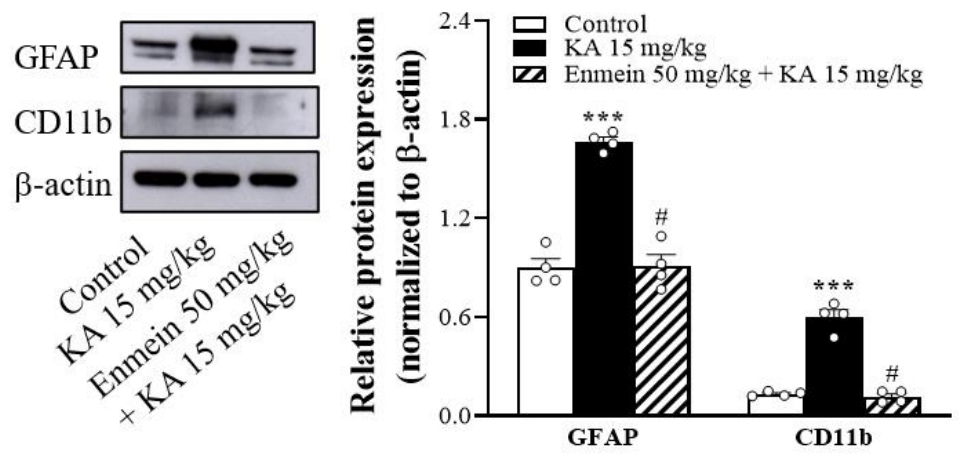

B

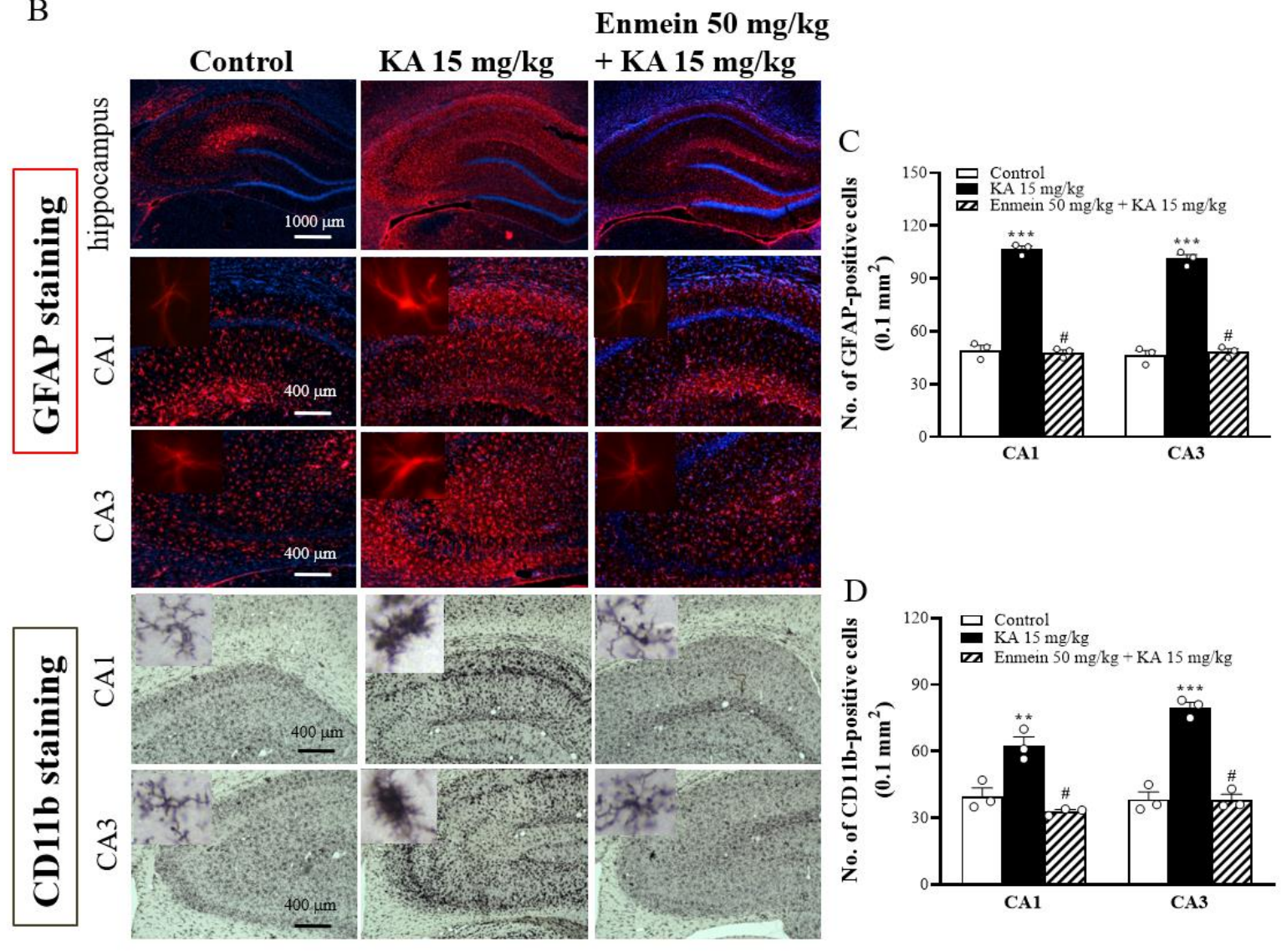

Figure 8. Effect of enmein on astrocyte and microglia activation in the hippocampus of rats with KA. (A) Western blot and semiquantitative analysis for the expression of GFAP and CD11b in the hippocampus at $72 \mathrm{~h}$ after KA injection. (B) Representative immunohistochemical mages of GFAP and CD11b staining at $72 \mathrm{~h}$ after i.p. KA. (C,D) Semiquantitative analysis of GFAP- and CD11b-positive neurons in the CA1 and CA3 regions. The insets are high magnification micrographs of astrocyte and microglial cells (Scale bar, $25 \mu \mathrm{m}$ ). Data are presented as mean \pm SEM. ( $n=4$ rats for each group). ${ }^{* *}, p<0.01$; $* * *, p<0.001$ vs. control group. \#, $p<0.05$ vs. KA group. 


\section{Discussion}

Excess glutamate is one of the main causes of neuronal death in neurological diseases [4]. Research on new drugs capable of reducing glutamate toxicity has received increasing attention. Among these, natural constituents from medicinal plants have been suggested to regulate the glutamate system and act as promising treatments for neurological disorders [13]. Diterpenoids are important and widely distributed natural compounds with various biological effects, including antitumor, anti-inflammatory, and immune modulation effects [32]. Enmein is a diterpenoid extracted from Isodon japonicus Hara that exhibits antitumor, anti-inflammatory, and immune modulation effects $[16,17,33]$. The present study provides the first description of the role of enmein in the central glutamate system, including its ability to regulate glutamate release from rat cerebrocortical nerve terminals and exhibit neuroprotective effects in a rat model of KA-induced glutamate excitotoxicity.

\subsection{Inhibition of Presynaptic Glutamate Release by Enmein}

The first relevant finding of this study was that enmein was able to inhibit the release of glutamate evoked by 4-AP depolarization of rat cerebrocortical nerve terminals. Concerning the cellular mechanism enmein-mediated inhibition of glutamate release, we considered two possibilities: (1) alteration of nerve terminal excitability and downstream regulation of $\mathrm{Ca}^{2+}$ influx into the terminal; and (2) direct regulation of exocytosis-coupled VDCCs that affect $\mathrm{Ca}^{2+}$ influx $[19,34]$. However, the first possibility was ruled out due to three observations. First, in the presence of $\mathrm{Ca}^{2+}$-free medium containing EGTA, enmein failed to significantly inhibit 4-AP-evoked glutamate release $\left(\mathrm{Ca}^{2+}\right.$-independent release), which depends solely on the membrane potential [35]. This indicates that enmein affects glutamate release by reducing 4-AP-evoked classical external $\mathrm{Ca}^{2+}$-dependent exocytosis. The vacuolar $\mathrm{H}^{+}$ATPase inhibitor bafilomycin A1, which causes glutamate depletion in synaptic vesicles, completely abolishes the inhibitory effect of enmein on 4-AP-evoked glutamate release, which supports this suggestion. Second, enmein had no effect the synaptosomal membrane potential, which was measured with a membrane potential-sensitive dye. Third, the inhibitory effect of enmein was observed when $\mathrm{KCl}$ was used as a depolarizing agent, indicating that the involvement of $\mathrm{K}^{+}$channels is not required [19]. These findings suggest that the inhibition of glutamate release by enmein is not a result of suppressing synaptosomal excitability. In addition, we demonstrated that enmein reduced the 4-AP-evoked increase in $\left[\mathrm{Ca}^{2+}\right]_{\mathrm{C}}$ using Fura-2. Moreover, the inhibitory effect of enmein on 4-AP-evoked glutamate release was completely prevented when release-coupled $\mathrm{N}$ - and $\mathrm{P} / \mathrm{Q}$-type $\mathrm{Ca}^{2+}$ channels were blocked. From the above results, we inferred that enmein suppresses evoked glutamate release through direct suppression of $\mathrm{N}$ - and $\mathrm{P} / \mathrm{Q}-\mathrm{type} \mathrm{Ca}^{2+}$ channels. However, whether enmein has a direct interaction with presynaptic VDCCs remains unknown. On the other hand, increased cytosolic $\mathrm{Ca}^{2+}$ in synaptic terminals can activate PKC to phosphorylate several proteins involved in exocytosis, including synapsin I. The phosphorylation of synapsin I causes cytoskeletal disassembly, which increases synaptic vesicle availability and glutamate release $[23,36]$. In the present study, we also found that the inhibitory action of enmein on 4-AP-evoked glutamate release was blocked by inhibiting PKC with the specific inhibitor GF109203X or Go6976. Furthermore, enmein significantly suppressed 4-AP-evoked PKC and its substrate synapsin I phosphorylation. These results, combined with the possibility that enmein effectively suppresses glutamate release components supported by $\mathrm{N}$ - and $\mathrm{P} / \mathrm{Q}$-type $\mathrm{Ca}^{2+}$ channels, suggest that enmein decreases $\mathrm{Ca}^{2+}$ influx through $\mathrm{N}$ - and $\mathrm{P} / \mathrm{Q}$-type $\mathrm{Ca}^{2+}$ channels, thereby reducing $\mathrm{PKC} /$ synapsin I phosphorylation and, as a result, reducing glutamate release from cerebrocortical synaptosomes.

\subsection{Prevention of Glutamate Excitotoxicity by Enmein}

The second relevant finding of this study was that enmein had a neuroprotective effect in a KA-induced glutamate excitotoxicity rat model. KA is a glutamate analog, and its neurotoxicity largely depends on enhancing the glutamate system, most likely through 
increasing glutamate receptor activation and glutamate release $[37,38]$. The systemic administration of KA in rats induces neuronal damage and gliosis in various brain regions, including the hippocampus, and these pathological phenomena resemble those seen in some neurological diseases [39]. Thus, KA is the most commonly used agent in animal models to test drugs for their potential neuroprotective properties [18]. In the present study, KA administration caused substantial neuronal death in the CA1 and CA3 regions of the hippocampus, as has been reported previously [37,40]. The administration of enmein before KA injection reduced KA-induced hippocampal neuronal death. The neuroprotective effect of enmein against KA-induced hippocampal damage could be linked to its effect on glutamate neurotransmission. Two lines of evidence support this notion. First, enmein pretreatment prevented KA-induced increases in glutamate levels in the hippocampus. Second, KA decreased the expression of EAAT2 and EAAT3 in the hippocampus; these effects were reversed by enmein pretreatment. EAAT2 and EAAT3 are glutamate transporters that take up excessive glutamate in the synaptic cleft and maintain glutamate homeostasis at excitatory synapses [24,41,42]. EAAT2 is highly expressed on astrocytes and accounts for more than $90 \%$ of brain glutamate uptake, whereas EAAT3 is mainly expressed on postsynaptic neurons throughout the brain [43]. Malfunction or aberrant expression of these glutamate transporters can result in excessive glutamate buildup, leading to excitotoxicity of neurons and involvement in the development of various neurological disorders $[26,44,45]$. Upregulation of EAAT2 and EAAT3 has been shown to suppress glutamate accumulation and limit excitotoxicity $[46,47]$. Thus, we infer that reduced glutamate concentrations caused by increasing glutamate uptake via glutamate transporters may explain enmein's neuroprotection against KA-induced excitotoxicity. In addition to increasing glutamate uptake via glutamate transporters, enmein's ability to block glutamate release from nerve terminals may help to explain its neuroprotective mechanism against KA-induced excitotoxicity in vivo. This hypothesis is based on the association between KA-induced neurotoxicity and excessive glutamate release $[37,38]$.

In addition, glutamate-induced neurotoxicity induces synaptic dysfunction, which leads to important neuropathological effects that have a considerable impact brain functionality and behavior [48]. In the present study, KA treatment in rats reduced the levels of synaptic marker proteins (synaptophysin and PSD95) in the hippocampus, and pretreatment with enmein reversed these effects. These proteins are essential for maintaining synaptic activity and plasticity [49]. Decreases in these proteins have been linked to KAinduced excitotoxic injury $[12,27,28]$. Therefore, we speculate that enmein could protect against KA-induced neuronal death by maintaining synaptophysin and PSD95 protein expression in the hippocampus.

Glutamate-induced excitotoxicity is also thought to be related to inflammatory processes [48]. Neuronal death and glutamate spillover from the synaptic region can both contribute to neuroinflammation $[50,51]$. Neuroinflammation, including the activation of microglia and astrocytes, is often associated with KA-induced excitotoxic injury [29-31]. Activated glial cells increase the production of proinflammatory cytokines and chemokines, which influence glutamate homeostasis by downregulating EAAT expression and upregulating glutamate receptors, thus increasing glutamatergic neurotransmission and excitotoxicity $[50,52]$. This evidence suggests that controlling KA-induced neuroinflammation is vital for protecting hippocampal neurons [30]. In the present study, we observed that KA substantially increased the levels of GFAP (an astrocytic marker) and CD11b (a microglial marker), as well as the number of activated microglia and astrocytes in the hippocampus; these increases were suppressed by enmein pretreatment. Thus, the suppression of neuroinflammation could be another mechanism that explains the neuroprotection exerted by enmein against KA-induced excitotoxicity. Our findings are consistent with those of previous studies that have reported the anti-inflammatory properties of enmein [16]. Accumulated evidence from animal models suggests that anti-inflammatory drugs may have potential neuroprotective effects for several neurological diseases. Although the mechanism by which enmein suppresses glial activation was not investigated in this study, 
toll-like receptors (TLRs) have been shown to play a critical role in glial cell activation and subsequent hippocampal neuron excitotoxicity [53]. Furthermore, preventing TLR activation suppresses glial activation and protects against excitotoxicity [53,54]. Whether enmein suppresses the activation of TLRs, thereby reducing glial cell activation and further suppressing KA-induced excitotoxic injuries, should be investigated in future studies. Taken together, our in vivo findings confirmed that enmein pretreatment improved hippocampal neuron protection in a rat model of excitotoxicity and suggested that the mechanism involves decreased glutamate levels, increased expression of EAAT2, EAAT3, synaptophysin and PSD95, and decreased glial cell activation.

\section{Materials and Methods}

\subsection{Animals}

Sprague-Dawley male rats (150-200 g) were kept in a controlled environment (12:12 h light-dark cycle, $24 \pm 1{ }^{\circ} \mathrm{C}, 55 \%$ relative humidity) with free access to food and water. All experimental protocols were designed with minimal animals. Experiments and animal use procedures were carried out in accordance with the National Institutes of Health Guide for the Care and Use of Laboratory Animals (NIH Publications No. 80-23, revised 1996) and approved by the Ethics Committee for Animal Research of the Fu Jen Catholic University (\#A10848). This study included 42 rats in total. Fresh brain tissue from 30 rats was used to measure glutamate release, $\mathrm{Ca}^{2+}$ concentration, and membrane potential, as well as for HPLC, and Western blotting. Histological staining was performed on fixed brain tissue from nine rats.

\subsection{Materials}

Enmein was purchased from Tauto Biotech (Shanghai, China) at 98\% purity. 4-AP, bafilomycin A1, GF109203X and Go6976 were purchased from Tocris Cookson (Bristol, UK). Fura-2-AM was purchased from Invitrogen (Carlsbad, CA, USA). $\omega$-CgTX GVIA and $\omega$-AgTX IVA were purchased from the Alomone lab (Jerusalem, Israel). PKC, PKC- $\alpha$, MARCKS, EAAT3, synaptophysin, GFAP and $\beta$-actin antibodies were obtained from Cell Signaling (Beverly, MA, USA). EAAT2, PSD95, CD11b p-PKC and p-PKC- $\alpha$, antibodies were obtained from Abcam (Cambridge, UK). NeuN antibody was obtained from Merck Millipore (Billerica, MA, USA). Horseradish peroxidase-conjugated secondary antibodies were obtained from Genetex (Zeeland, MI, USA). Alexa Fluor 488 and DyLight 594 were obtained from Invitrogen (Waltham, MA, USA). KA, DMSO, Cresyl violet, 4' ,6-diamidino2-phenylindole (DAPI) and general reagents were purchased from Sigma Chemical Co. (St Louis, MO, USA).

\subsection{Preparation of Synaptosomes}

Synaptosomes were prepared as previously reported [55,56]. Briefly, rats were killed by cervical dislocation and decapitation. The cerebral cortex was rapidly removed and homogenized in an ice-cold HEPES-buffered medium containing $0.32 \mathrm{M}$ sucrose ( $\mathrm{pH}$ 7.4). The homogenate was centrifuged at $3000 \times g$ for $10 \mathrm{~min}$ at $4{ }^{\circ} \mathrm{C}$. The supernatant was retained and centrifuged at $14,500 \times g$ for $12 \mathrm{~min}$ at $4{ }^{\circ} \mathrm{C}$. The pellet was resuspended and layered on top of a discontinuous Percoll gradient before being centrifuged at 32,500 $\mathrm{g}$ for $7 \mathrm{~min}$ at $4{ }^{\circ} \mathrm{C}$. The protein concentration was determined using the Bradford assay. The synaptosomes were centrifuged in the final wash to obtain synaptosomal pellets containing $0.5 \mathrm{mg}$ protein.

\subsection{Determination of Glutamate Release}

Synaptosomal pellets were analyzed for glutamate release using an enzyme-linked fluorescence method as previously described $[55,57]$. In brief, synaptosomes were suspended in HEPES-buffered medium containing $2 \mathrm{mM} \mathrm{NADP}^{+}, 50$ units of glutamate dehydrogenase, and $1.2 \mathrm{mM} \mathrm{CaCl}_{2}$, and the synaptosome suspension was stimulated with either $1 \mathrm{mM} 4$-AP or $15 \mathrm{mM} \mathrm{KCl}$ after $5 \mathrm{~min}$. Fluorescence increases due to NADPH production 
were measured $(\lambda$ excitation $=340 \mathrm{~nm}$ and $\lambda$ emission $=460 \mathrm{~nm}$ ) with a PerkinElmer LS55 spectrofluorimeter. The amount of glutamate released was calibrated by a standard of exogenous glutamate $(5 \mathrm{nmol})$ and expressed as nanomoles glutamate per milligram synaptosomal protein (nmol/mg). The values given in the text and depicted in the bar graphs represent the levels of glutamate cumulatively released after $5 \mathrm{~min}$ of depolarization and are expressed as $\mathrm{nmol} / \mathrm{mg} / 5 \mathrm{~min}$.

\subsection{Determination of Exocytosis}

Exocytosis was measured with an FM1-43 dye. FM1-43 is a fluorescent styryl dye that is lipophilic but not membrane permeable. When the styryl dye FM1-43 reversibly partitions into the outer leaflet of the exposed plasma membrane, its fluorescence increases. FM1-43 dye can be taken up into synaptic vesicles by endocytosis, and during subsequent exocytosis, FM1-43 is lost to the extracellular medium, resulting in a decrease in fluorescence [20]. Briefly, the synaptosomes were loaded with FM1-43 (100 $\mu \mathrm{M})$ for $60 \mathrm{~s}$ before being loaded with extracellular high $\mathrm{K}^{+}(30 \mathrm{mM})$ for $90 \mathrm{~s}$ at room temperature. Then, the synaptosomes were washed with HEPES-buffered medium and centrifuged for $1 \mathrm{~min}$ at $3000 \times g$, and the synaptosome suspension was stimulated with $1 \mathrm{mM} 4$-AP. The release of accumulated FM1-43 was measured as the decrease in fluorescence upon releasing dye into the solution $(\lambda$ excitation $=488 \mathrm{~nm}$ and $\lambda$ emission $=540 \mathrm{~nm}$ ).

\subsection{Determination of Cytosolic Free $\mathrm{Ca}^{2+}$ Concentration $\left(\left[\mathrm{Ca}^{2+}\right]_{C}\right)$}

$\left[\mathrm{Ca}^{2+}\right]_{C}$ measurement was determined by the fluorescent probe Fura-2-AM. The synaptosomes were incubated with $5 \mu \mathrm{M}$ Fura-2 in a HEPES-buffered medium containing $0.1 \mathrm{mM} \mathrm{CaCl} 2$ for $30 \mathrm{~min}$ at $37^{\circ} \mathrm{C}$. After Fura-2 loading, the synaptosomes were centrifuged for $1 \mathrm{~min}$ at $3000 \times g$, and the pellets were resuspended in HEPES-buffered medium containing $1.2 \mathrm{mM} \mathrm{CaCl}_{2}$. Fura-2/Ca fluorescence was measured with a PerkinElmer LS55 spectrofluorimeter at $340 \mathrm{~nm}$ and $510 \mathrm{~nm}$. $\left[\mathrm{Ca}^{2+}\right]_{C}(\mathrm{nM})$ was calculated using the equations described previously [58].

\subsection{Determination of Synaptosomal Membrane Potential}

The synaptosomal membrane potential was detected using the positively charged membrane potential-sensitive carbocyanine dye $\operatorname{DiSC}_{3}(5)$ [59]. $\operatorname{DiSC}_{3}(5)$ fluorescence was measured $(\lambda$ excitation $=646 \mathrm{~nm}$ and $\lambda$ emission $=674 \mathrm{~nm})$ with a PerkinElmer LS55 spectrofluorimeter. Data are expressed in fluorescence units.

\subsection{Western Blot}

Western blotting was performed as previously described [60]. Briefly, $30 \mu \mathrm{g}$ of protein was loaded on a $10 \%$ sodium dodecyl sulfate (SDS)-polyacrylamide gel electrophoresis. After separation, the protein was blotted onto a nitrocellulose membrane and blocked for $1 \mathrm{~h}$ with 5\% skim milk. The membrane was incubated with a specific primary antibody and a horseradish peroxidase-conjugated secondary antibody (1:5000) using enhanced chemiluminescence (ECL; Amersham, Buckinghamshire, UK). The primary antibodies used in this study included PKC (1:700), p-PKC (1:2000), PKC- $\alpha$ (1:600), p-PKC- $\alpha$ (1:2000), MARCKS (1:250), NeuN (1:5000), EAAT2 (1:50,000), EAAT3 (1:10,000), synaptophysin (1:50,000), PSD95 (1:1000), GFAP (1:5000), CD11b (1:9000), and $\beta$-actin (1:1000). Scanning densitometry was used to quantify four to five independent experiments, which were then analyzed with ImageJ software (Synoptics, Cambridge, UK).

\subsection{Immunohistochemistry}

The rats were divided into three groups at random: the DMSO-treated group (control), the KA-treated group, and the enmein $50 \mathrm{mg} / \mathrm{kg}+\mathrm{KA}$-treated group. Enmein was dissolved in a saline solution containing 1\% DMSO and administered (i.p.) 30 min before $\mathrm{KA}$ injection (15 mg/ $\mathrm{kg}$ in $0.9 \% \mathrm{NaCl}, \mathrm{pH} 7.0$, i.p.). Seventy-two hours after KA injection, the rats were euthanized with $\mathrm{CO}_{2}$ and fixed by transcardial perfusion with $4 \%$ buffered 
paraformaldehyde ( $\mathrm{pH}$ 7.2). The brains were removed and embedded in paraffin after fixation in $4 \%$ buffered paraformaldehyde, followed by $0.1 \mathrm{M}$ phosphate-buffered saline ( $\mathrm{pH}$ 7.2) for $24 \mathrm{~h}$ at $4{ }^{\circ} \mathrm{C}$. A sliding microtome was used to acquire $25-\mu \mathrm{m}$-thick coronal brain sections from the hippocampus. For Nissl staining, the sections were mounted on a glass slide and stained with $0.1 \%$ aqueous Cresyl violet stain for $20 \mathrm{~min}$ after dehydration in graded ethanol solutions. For NeuN and GFAP staining, the sections were blocked in phosphate buffered saline containing $10 \%$ normal goat serum for $1 \mathrm{~h}$ to block nonspecific antibody binding before being incubated overnight at $4{ }^{\circ} \mathrm{C}$ with anti-NeuN antibody (1:500) or anti-GFAP antibody (1:1000) and subsequently incubated with IgG-DyLight 594 (1:1000) and FITC-conjugated IgG (1:1000) for $2 \mathrm{~h}$ at $25^{\circ} \mathrm{C}$. Nuclei were counterstained with DAPI $(50 \mathrm{ng} / \mathrm{mL})$. For CD11b staining, the sections were incubated overnight with anti-CD11b antibody (1:500). The sections were then incubated with goat biotinylated anti-mouse secondary antibodies (1:200; Vector Laboratories, Burlingame, CA, USA) for $2 \mathrm{~h}$ and subsequently incubated with ExtrAvidin peroxidase (1:1000) for $1 \mathrm{~h}$ at room temperature. After rinsing in $0.1 \mathrm{M}$ phosphate-buffered saline for $20 \mathrm{~min}$, the sections were reacted with 0.025\% 3,3-diaminobenzidine tetrahydrochloride (DAB) solution in a phosphate-buffered saline containing $0.0025 \%$ hydrogen peroxide for $6 \mathrm{~min}$. Immunostaining images were captured by a digital camera (Leica, Wetzlar, Germany) attached to a fluorescence microscope (Zeiss Axioskop 40, Göttingen, Lower Saxony, Germany). Leica $4 \times, 10 \times$ or $40 \times$ objective lenses with numerical apertures (NA) of 0.1 or 0.25 were used in this study. The number of surviving neurons and NeuN-, GFAP-, or CD11b-positive cells were counted in a $255 \mu \mathrm{m} \times 255 \mu \mathrm{m}$ region of the hippocampal CA1/CA3 using ImageJ software (Synoptics, Cambridge, UK).

\subsection{Glutamate Levels in Brain Tissue from High-Performance Liquid Chromatography (HPLC)}

As previously reported [11], rats were killed by cervical dislocation and decapitation, and the hippocampus was rapidly dissected $72 \mathrm{~h}$ after KA injection. Fifty milligrams of hippocampal tissue were homogenized with $200 \mu \mathrm{L}$ distilled water and centrifuged for $10 \mathrm{~min}$ at $1500 \times g\left(4^{\circ} \mathrm{C}\right)$. The supernatant was filtered through $0.22 \mu \mathrm{m}$ filters, and then $8 \mu \mathrm{L}$ of the solution was injected into an HPLC system with electrochemical detection (HTEC500, Eicom, Kyoto, Japan). The relative free glutamate concentration was determined based on peak areas by an external standard method.

\subsection{Statistical Analysis}

The mean \pm standard error of the mean (SEM) was calculated. Statistical significance $(p<0.05)$ among groups was determined by Student's $t$ tests or one-way analysis of variance (ANOVA) followed by Tukey's multiple comparisons test using GraphPad Prism software (La Jolla, CA, USA).

\section{Conclusions}

Our results show for the first time the glutamate release-inhibiting and anti-excitotoxic properties of enmein, a diterpenoid extracted from Isodon japonicus Hara. We demonstrate that enmein inhibits presynaptic glutamate release by suppressing $\mathrm{Ca}^{2+}$ influx and the PKC-dependent pathway. Furthermore, enmein prevents KA-induced glutamate excitotoxicity in vivo by suppressing gliosis and reducing glutamate accumulation via increased glutamate uptake. Our study may provide a pharmacological basis for the clinical use of enmein in developing new drugs for central nervous system diseases that involve glutamate excitotoxicity.

Author Contributions: Conceptualization, C.-W.L., Y.-C.H., T.-Y.L. and S.-J.W.; data curation, C.-W.L., Y.-C.H., K.-M.C., M.-Y.L. and T.-Y.L.; funding acquisition, C.-W.L., T.-Y.L. and S.-J.W.; investigation, C.-W.L., Y.-C.H., K.-M.C., T.-Y.L. and S.-J.W.; supervision, S.-J.W.; writing—original draft, S.-J.W.; writing - review \& editing, M.-Y.L. and S.-J.W. All authors have read and agreed to the published version of the manuscript. 
Funding: This research was funded by the Ministry of Science and Technology (MOST 110-2320-B030-009) and the Far Eastern Memorial Hospital (FEMH-2021-C-007; FEMH-2021-C-010), Taiwan.

Institutional Review Board Statement: The study was conducted according to the guidelines of the Care and Use of Laboratory Animals and approved by the Fu Jen Institutional Animal Care and Utilization Committee (No. A10848, 5 March, 2020).

Informed Consent Statement: Not applicable.

Data Availability Statement: The data presented in this study are available on request from the corresponding author.

Conflicts of Interest: The authors declare no conflict of interest.

\section{References}

1. Headley, P.M.; Grillner, S. Excitatory amino acids and synaptic transmission: The evidence for a physiological function. Trends Pharmacol. Sci. 1990, 11, 205-211. [CrossRef]

2. Javitt, D.C. Glutamate as a therapeutic target in psychiatric disorders. Mol. Psychiatry 2004, 9, 984-997, 979. [CrossRef]

3. Meldrum, B.; Garthwaite, J. Excitatory amino acid neurotoxicity and neurodegenerative disease. Trends Pharmacol. Sci. 1990, 11, 379-387. [CrossRef]

4. Dong, X.X.; Wang, Y.; Qin, Z.H. Molecular mechanisms of excitotoxicity and their relevance to pathogenesis of neurodegenerative diseases. Acta Pharmacol. Sin. 2009, 30, 379-387. [CrossRef]

5. González, J.C.; Egea, J.; Del Carmen Godino, M.; Fernandez-Gomez, F.J.; Sánchez-Prieto, J.; Gandía, L.; García, A.G.; Jordán, J.; Hernández-Guijo, J.M. Neuroprotectant minocycline depresses glutamatergic neurotransmission and Ca ${ }^{2+}$ signalling in hippocampal neurons. Eur. J. Neurosci. 2007, 26, 2481-2495. [CrossRef]

6. Lazarevic, V.; Yang, Y.; Ivanova, D.; Fejtova, A.; Svenningsson, P. Riluzole attenuates the efficacy of glutamatergic transmission by interfering with the size of the readily releasable neurotransmitter pool. Neuropharmacology 2018, 143, 38-48. [CrossRef] [PubMed]

7. Xu, H.; Zhao, B.; She, Y.; Song, X. Dexmedetomidine ameliorates lidocaine-induced spinal neurotoxicity via inhibiting glutamate release and the PKC pathway. Neurotoxicology 2018, 69, 77-83. [CrossRef] [PubMed]

8. Rodrigues, T.; Reker, D.; Schneider, P.; Schneider, G. Counting on natural products for drug design. Nat. Chem. $2016,8,531-541$. [CrossRef]

9. Chang, Y.; Lu, C.W.; Lin, T.Y.; Huang, S.K.; Wang, S.J. Baicalein, a Constituent of Scutellaria baicalensis, Reduces Glutamate Release and Protects Neuronal Cell Against Kainic Acid-Induced Excitotoxicity in Rats. Am. J. Chin. Med. 2016, 44, 943-962. [CrossRef]

10. Lu, C.W.; Hsieh, H.L.; Lin, T.Y.; Hsieh, T.Y.; Huang, S.K.; Wang, S.J. Echinacoside, an Active Constituent of Cistanche Herba, Exerts a Neuroprotective Effect in a Kainic Acid Rat Model by Inhibiting Inflammatory Processes and Activating the Akt/GSK3 $\beta$ Pathway. Biol. Pharm. Bull. 2018, 41, 1685-1693. [CrossRef]

11. Lu, C.W.; Lin, T.Y.; Wang, S.J. 11-Keto- $\beta$-Boswellic Acid Attenuates Glutamate Release and Kainic Acid-Induced Excitotoxicity in the Rat Hippocampus. Planta Med. 2020, 86, 434-441. [CrossRef]

12. Lu, C.W.; Lin, T.Y.; Pan, T.L.; Wang, P.W.; Chiu, K.M.; Lee, M.Y.; Wang, S.J. Asiatic Acid Prevents Cognitive Deficits by Inhibiting Calpain Activation and Preserving Synaptic and Mitochondrial Function in Rats with Kainic Acid-Induced Seizure. Biomedicines 2021, 9, 284. [CrossRef]

13. Liu, Y.; Wang, S.; Kan, J.; Zhang, J.; Zhou, L.; Huang, Y.; Zhang, Y. Chinese Herbal Medicine Interventions in Neurological Disorder Therapeutics by Regulating Glutamate Signaling. Curr. Neuropharmacol. 2020, 18, 260-276. [CrossRef] [PubMed]

14. Li, B.; Tian, X. Enmein type diterpenoids from Isodon japonica. Phytochemistry 2001, 58, 543-546. [CrossRef]

15. Xu, S.; Pei, L.; Li, D.; Yao, H.; Cai, H.; Yao, H.; Wu, X.; Xu, J. Synthesis and antimycobacterial evaluation of natural oridonin and its enmein-type derivatives. Fitoterapia 2014, 99, 300-306. [CrossRef] [PubMed]

16. Li, H.; Sun, B.; Wang, M.; Hu, X.; Gao, X.; Xu, S.; Xu, Y.; Xu, J.; Hua, H.; Li, D. Bioactive enmein-type 6,7-seco-ent-kaurane diterpenoids: Natural products, synthetic derivatives and apoptosis related mechanism. Arch. Pharm. Res. 2018, 41, $1051-1061$. [CrossRef]

17. Li, D.; Hu, X.; Han, T.; Liao, J.; Xiao, W.; Xu, S.; Li, Z.; Wang, Z.; Hua, H.; Xu, J. NO-Releasing Enmein-Type Diterpenoid Derivatives with Selective Antiproliferative Activity and Effects on Apoptosis-Related Proteins. Molecules 2016, 21, 1193. [CrossRef] [PubMed]

18. Wang, Q.; Yu, S.; Simonyi, A.; Sun, G.Y.; Sun, A.Y. Kainic acid-mediated excitotoxicity as a model for neurodegeneration. Mol. Neurobiol. 2005, 31, 3-16. [CrossRef]

19. Nicholls, D.G. Presynaptic modulation of glutamate release. Prog. Brain Res. 1998, 116, 15-22. [CrossRef]

20. Baldwin, M.L.; Rostas, J.A.; Sim, A.T. Two modes of exocytosis from synaptosomes are differentially regulated by protein phosphatase types 2A and 2B. J. Neurochem. 2003, 85, 1190-1199. [CrossRef]

21. Vázquez, E.; Sánchez-Prieto, J. Presynaptic modulation of glutamate release targets different calcium channels in rat cerebrocortical nerve terminals. Eur. J. Neurosci. 1997, 9, 2009-2018. [CrossRef] [PubMed]

22. Barrie, A.P.; Nicholls, D.G.; Sanchez-Prieto, J.; Sihra, T.S. An ion channel locus for the protein kinase C potentiation of transmitter glutamate release from guinea pig cerebrocortical synaptosomes. J. Neurochem. 1991, 57, 1398-1404. [CrossRef] 
23. Coffey, E.T.; Sihra, T.S.; Nicholls, D.G.; Pocock, J.M. Phosphorylation of synapsin I and MARCKS in nerve terminals is mediated by $\mathrm{Ca}^{2+}$ entry via an Aga-GI sensitive $\mathrm{Ca}^{2+}$ channel which is coupled to glutamate exocytosis. FEBS Lett. 1994, 353, 264-268. [CrossRef]

24. Beart, P.M.; O'Shea, R.D. Transporters for L-glutamate: An update on their molecular pharmacology and pathological involvement. Br. J. Pharmacol. 2007, 150, 5-17. [CrossRef]

25. Masliah, E.; Raber, J.; Alford, M.; Mallory, M.; Mattson, M.P.; Yang, D.; Wong, D.; Mucke, L. Amyloid protein precursor stimulates excitatory amino acid transport. Implications for roles in neuroprotection and pathogenesis. J. Biol. Chem. 1998, 273, 12548-12554. [CrossRef]

26. Sheldon, A.L.; Robinson, M.B. The role of glutamate transporters in neurodegenerative diseases and potential opportunities for intervention. Neurochem. Int. 2007, 51, 333-355. [CrossRef]

27. Zhang, F.X.; Sun, Q.J.; Zheng, X.Y.; Lin, Y.T.; Shang, W.; Wang, A.H.; Duan, R.S.; Chi, Z.F. Abnormal expression of synaptophysin, SNAP-25, and synaptotagmin 1 in the hippocampus of kainic acid-exposed rats with behavioral deficits. Cell. Mol. Neurobiol. 2014, 34, 813-824. [CrossRef]

28. Zhu, X.; Liu, J.; Huang, S.; Zhu, W.; Wang, Y.; Chen, O.; Xue, J. Neuroprotective effects of isoliquiritigenin against cognitive impairment via suppression of synaptic dysfunction, neuronal injury, and neuroinflammation in rats with kainic acid-induced seizures. Int. Immunopharmacol. 2019, 72, 358-366. [CrossRef]

29. Penkowa, M.; Florit, S.; Giralt, M.; Quintana, A.; Molinero, A.; Carrasco, J.; Hidalgo, J. Metallothionein reduces central nervous system inflammation, neurodegeneration, and cell death following kainic acid-induced epileptic seizures. J. Neurosci. Res. 2005, 79, 522-534. [CrossRef] [PubMed]

30. Avignone, E.; Ulmann, L.; Levavasseur, F.; Rassendren, F.; Audinat, E. Status epilepticus induces a particular microglial activation state characterized by enhanced purinergic signaling. J. Neurosci. 2008, 28, 9133-9144. [CrossRef] [PubMed]

31. Choi, H.S.; Park, J.H.; Ahn, J.H.; Hong, S.; Cho, J.H.; Won, M.H.; Lee, C.H. The anti-inflammatory activity of duloxetine, a serotonin/norepinephrine reuptake inhibitor, prevents kainic acid-induced hippocampal neuronal death in mice. J. Neurol. Sci. 2015, 358, 390-397. [CrossRef]

32. De Sousa, I.P.; Sousa Teixeira, M.V.; Jacometti Cardoso Furtado, N.A. An Overview of Biotransformation and Toxicity of Diterpenes. Molecules 2018, 23, 1387. [CrossRef]

33. Zhang, Y.; Liu, J.; Jia, W.; Zhao, A.; Li, T. Distinct immunosuppressive effect by Isodon serra extracts. Int. Immunopharmacol. 2005, 5, 1957-1965. [CrossRef] [PubMed]

34. Wu, L.G.; Saggau, P. Presynaptic inhibition of elicited neurotransmitter release. Trends Neurosci. 1997, 20, 204-212. [CrossRef]

35. Nicholls, D.G.; Sihra, T.S.; Sanchez-Prieto, J. Calcium-dependent and -independent release of glutamate from synaptosomes monitored by continuous fluorometry. J. Neurochem. 1987, 49, 50-57. [CrossRef]

36. Nichols, R.A.; Sihra, T.S.; Czernik, A.J.; Nairn, A.C.; Greengard, P. Calcium/calmodulin-dependent protein kinase II increases glutamate and noradrenaline release from synaptosomes. Nature 1990, 343, 647-651. [CrossRef]

37. Ferkany, J.W.; Zaczek, R.; Coyle, J.T. Kainic acid stimulates excitatory amino acid neurotransmitter release at presynaptic receptors. Nature 1982, 298, 757-759. [CrossRef]

38. Chittajallu, R.; Vignes, M.; Dev, K.K.; Barnes, J.M.; Collingridge, G.L.; Henley, J.M. Regulation of glutamate release by presynaptic kainate receptors in the hippocampus. Nature 1996, 379, 78-81. [CrossRef]

39. Ben-Ari, Y. Limbic seizure and brain damage produced by kainic acid: Mechanisms and relevance to human temporal lobe epilepsy. Neuroscience 1985, 14, 375-403. [CrossRef]

40. Smani, D.; Sarkar, S.; Raymick, J.; Kanungo, J.; Paule, M.G.; Gu, Q. Downregulation of 14-3-3 Proteins in a Kainic Acid-Induced Neurotoxicity Model. Mol. Neurobiol. 2018, 55, 122-129. [CrossRef] [PubMed]

41. Rose, C.R.; Felix, L.; Zeug, A.; Dietrich, D.; Reiner, A.; Henneberger, C. Astroglial Glutamate Signaling and Uptake in the Hippocampus. Front. Mol. Neurosci. 2017, 10, 451. [CrossRef]

42. Hayashi, M.K. Structure-Function Relationship of Transporters in the Glutamate-Glutamine Cycle of the Central Nervous System. Int. J. Mol. Sci. 2018, 19, 1177. [CrossRef]

43. Rothstein, J.D.; Martin, L.; Levey, A.I.; Dykes-Hoberg, M.; Jin, L.; Wu, D.; Nash, N.; Kuncl, R.W. Localization of neuronal and glial glutamate transporters. Neuron 1994, 13, 713-725. [CrossRef]

44. Takahashi, K.; Foster, J.B.; Lin, C.L. Glutamate transporter EAAT2: Regulation, function, and potential as a therapeutic target for neurological and psychiatric disease. Cell. Mol. Life Sci. 2015, 72, 3489-3506. [CrossRef]

45. Pajarillo, E.; Rizor, A.; Lee, J.; Aschner, M.; Lee, E. The role of astrocytic glutamate transporters GLT-1 and GLAST in neurological disorders: Potential targets for neurotherapeutics. Neuropharmacology 2019, 161, 107559. [CrossRef] [PubMed]

46. Nematipour, S.; Vahidinia, Z.; Nejati, M.; Naderian, H.; Beyer, C.; Azami Tameh, A. Estrogen and progesterone attenuate glutamate neurotoxicity via regulation of EAAT3 and GLT-1 in a rat model of ischemic stroke. Iran. J. Basic Med. Sci. 2020, 23, 1346-1352. [CrossRef] [PubMed]

47. Green, J.L.; Dos Santos, W.F.; Fontana, A.C.K. Role of glutamate excitotoxicity and glutamate transporter EAAT2 in epilepsy: Opportunities for novel therapeutics development. Biochem. Pharmacol. 2021, 193, 114786. [CrossRef] [PubMed]

48. Iovino, L.; Tremblay, M.E.; Civiero, L. Glutamate-induced excitotoxicity in Parkinson's disease: The role of glial cells. J. Pharmacol. Sci. 2020, 144, 151-164. [CrossRef] 
49. Martin, S.J.; Grimwood, P.D.; Morris, R.G. Synaptic plasticity and memory: An evaluation of the hypothesis. Annu. Rev. Neurosci. 2000, 23, 649-711. [CrossRef]

50. Haroon, E.; Miller, A.H.; Sanacora, G. Inflammation, Glutamate, and Glia: A Trio of Trouble in Mood Disorders. Neuropsychopharmacology 2017, 42, 193-215. [CrossRef]

51. Tilleux, S.; Hermans, E. Neuroinflammation and regulation of glial glutamate uptake in neurological disorders. J. Neurosci. Res. 2007, 85, 2059-2070. [CrossRef] [PubMed]

52. Skaper, S.D.; Facci, L.; Zusso, M.; Giusti, P. An Inflammation-Centric View of Neurological Disease: Beyond the Neuron. Front. Cell. Neurosci. 2018, 12, 72. [CrossRef] [PubMed]

53. Hong, J.; Cho, I.H.; Kwak, K.I.; Suh, E.C.; Seo, J.; Min, H.J.; Choi, S.Y.; Kim, C.H.; Park, S.H.; Jo, E.K.; et al. Microglial Tolllike receptor 2 contributes to kainic acid-induced glial activation and hippocampal neuronal cell death. J. Biol. Chem. 2010, 285, 39447-39457. [CrossRef]

54. Alomar, S.Y.; Barakat, M.B.; Eldosoky, M.; Atef, H.; Mohamed, A.S.; Elhawary, R.; El-Shafey, M.; Youssef, A.M.; Elkazaz, A.Y.; Gabr, A.M.; et al. Protective effect of metformin on rat diabetic retinopathy involves suppression of toll-like receptor $4 /$ nuclear factor-k B expression and glutamate excitotoxicity. Int. Immunopharmacol. 2021, 90, 107193. [CrossRef] [PubMed]

55. Nicholls, D.G.; Sihra, T.S. Synaptosomes possess an exocytotic pool of glutamate. Nature 1986, 321, 772-773. [CrossRef] [PubMed]

56. Lu, C.W.; Lin, T.Y.; Huang, S.K.; Wang, S.J. Echinacoside Inhibits Glutamate Release by Suppressing Voltage-Dependent Ca(2+) Entry and Protein Kinase C in Rat Cerebrocortical Nerve Terminals. Int. J. Mol. Sci. 2016, 17, 1006. [CrossRef]

57. Lin, T.-Y.; Lu, C.-W.; Wu, C.-C.; Huang, S.-K.; Wang, S.-J. Palmitoylethanolamide Inhibits Glutamate Release in Rat Cerebrocortical Nerve Terminals. Int. J. Mol. Sci. 2015, 16, 5555-5571. [CrossRef]

58. Grynkiewicz, G.; Poenie, M.; Tsien, R.Y. A new generation of $\mathrm{Ca}^{2+}$ indicators with greatly improved fluorescence properties. J. Biol. Chem. 1985, 260, 3440-3450. [CrossRef]

59. Akerman, K.E.; Scott, I.G.; Heikkilä, J.E.; Heinonen, E. Ionic dependence of membrane potential and glutamate receptor-linked responses in synaptoneurosomes as measured with a cyanine dye, DiS-C2-(5). J. Neurochem. 1987, 48, 552-559. [CrossRef]

60. Hung, Y.-C.; Kuo, Y.-H.; Hsieh, P.-W.; Hsieh, T.-Y.; Kuo, J.-R.; Wang, S.-J. Chlorogenic Acid Decreases Glutamate Release from Rat Cortical Nerve Terminals by P/Q-Type $\mathrm{Ca}^{2+}$ Channel Suppression: A Possible Neuroprotective Mechanism. Int. J. Mol. Sci. 2021, 22, 11447. [CrossRef] 